\title{
Author Correction: A global effort to dissect the human genetic basis of resistance to SARS-CoV-2 infection
}

Evangelos Andreakos DD, Laurent Abel, Donald C. Vinh (D), Elżbieta Kaja Di, Beth A. Drolet, Qian Zhang, Cliona O'Farrelly, Giuseppe Novelli, Carlos Rodríguez-Gallego, Filomeen Haerynck, Carolina Prando D, Aurora Pujol, COVID Human Genetic Effort*, Helen C. Su, Jean-Laurent Casanova and András N. Spaan (D)

Correction to: Nature Immunology https://doi.org/10.1038/s41590-021-01030-z, published online 18 October 2021.

In the version of the article originally published, the list of collaborators with the COVID Human Genetic Effort was incomplete. The list has now been amended to include both Carlos Flores (Research Unit, Hospital Universitario Nuestra Señora de Candelaria, Santa Cruz de Tenerife, Spain; CIBER de Enfermedades Respiratorias, Instituto de Salud Carlos III, Madrid, Spain; Genomics Division, Instituto Tecnológico y de Energías Renovables (ITER), Santa Cruz de Tenerife, Spain) and Rebeca Pérez de Diego (Laboratory of Immunogenetics of Human Diseases, Innate Immunity Group, IdiPAZ Institute for Health Research, La Paz Hospital, Madrid, Spain; Interdepartmental Group of Immunodeficiencies, Madrid, Spain). The changes have been made to the online version of the article.

${ }^{*}$ A list of members and their affiliations appears at the end of the paper.

Published online: 24 November 2021

https://doi.org/10.1038/s41590-021-01096-9

(c) Springer Nature America, Inc. 2021

\section{Author Correction: Interfer(on)-ing with vascular repair after acute brain injury}

Jeremy R. Herrmann and Dennis W. Simon (1)

Correction to: Nature Immunology https://doi.org/10.1038/s41590-021-01034-9, published online 23 September 2021.

In the version of this article initially published, an Acknowledgements section was missing. The following has now been included: “This work was supported by the National Institutes of Health: grant nos. T32HD040686 (J.R.H.) and R21NS115174 (D.W.S.).”

Published online: 11 January 2022

https://doi.org/10.1038/s41590-022-01128-y

() Springer Nature America, Inc. 2022 